\title{
New insight into RBC membrane and trypanosome infection
}

\begin{abstract}
Natural herbs and several synthetic drugs had been used to treat trypanosomiasis and are usually directed to kill the trypanosome. However, Red Blood cells (RBCs) membranes that is the first infection site for trypanosome remains defenseless to the invasion. Hence, there is need to protect the membrane against the invasion to limit the gravity of first acute parasitaemia before immune system is ready to defend the body against the parasites.
\end{abstract}

Keywords: RBCs, membrane, trypanosomiasis, parasitaemia, immune system, haematological changes, hemoglobin, glycolipids
Volume 8 Issue 5 - 2019

\author{
Olanrewaju Roland Akinseye, Adelabu \\ Mustapha, Otunla Moses \\ Department of vector and parasitology, Nigerian Institute for \\ Trypanosomiasis (\& Onchocerciasis) Research, Nigeria
}

\begin{abstract}
Correspondence: Olanrewaju Roland Akinseye,
Department of vector and parasitology, Nigerian Institute for

Trypanosomiasis (\& Onchocerciasis) Research, Nigeria,

Tel +234703 I828325, Email akinseyeroland@gmail.com
\end{abstract}

Received: August 19, 2019 | Published:September II, 2019
Abbreviations: RBC, red blood cell; PCV, packed cell volume; PAS, periodic acid-Schiff's; NAIs, neuraminidase inhibitors; GP, glycoproteins; GL, glycolipids

\section{Introduction}

Trypanosomiasis is an endemic disease which is invariably fatal if not treated. It is characterized by biochemical and haematological changes which often influence the pathogenesis of the disease. The disease is connected with a rapid decrease $\mathrm{RBC}$ counts, haemoglobin $(\mathrm{Hb})$ concentration and packed cell volume (PCV) and unhealthy pale appearance in the mucosa membranes in the infected hosts. ${ }^{1}$ Red blood cell membrane is a bilayer which consists of proteins, glycolipids and unique molecules and structures which confer distinct properties associated with it. The erythrocytes of humans, form the important cells generally employed in the investigation of biological membranes, have some glycoproteins in their cell membrane. ${ }^{2}$

These membrane proteins are band 3 and glycophorins A-D, and some others glycoproteins. Glycophorin A which is an important constituent of RBC membrane glycoproteins is a sialic acid conjugate with oligosaccharide. ${ }^{3}$ The event through which red blood cells become adhere to trypanosome has been demonstrated in vitro in $T$. gambiense, T. rhodesiense, T. congolense, T. brucei, T. lewisi and $T$. evansi infections. ${ }^{4,5}$ The process of adhesion can lead to haemolysis of red cells (otherwise known as anaemia) which may be caused by the haemolytic factors such as haemolysin or high concentration FFA (such as linolenic acid) introduced into the red cells by the parasites. The haemolysis of red cells has been reported in $T$. congolense incubated red cells. ${ }^{4}$ The trypanosomes adhere to $\mathrm{RBCs}$ create breaches or holes through which cellular constituents leak into the extracellular space, thereby result in the abnormalities in the level of these biochemical and haematogical parameters. RBCs cell membrane posed mechanical resistance to the trypanosomes before they are able to adhere successfully to the membrane and cause damages but the resistance is not enough. The existence of many pathological form or shape assumed by RBC during the infection which were confirmed by transmission and scanning electron microscopy, ${ }^{4}$ depict the extent of infection of the parasites on RBCs.
Hence, the essence of this review is to suggest means to fortify and protect red blood cell membrane before the application of the necessary medications as it is the centre which receives first attack that lead into massive destruction of the red cells.

\section{Red cell membrane}

In Figure 1 the cell membrane of a red blood cell, like all other cell membranes, consists of a two layers of lipid known as bilayer which are stable and the membrane is equipped intracellularly and extracellularly with proteins, glycolipids and unique molecules and structures which confer distinct properties associated with it. The erythrocytes of humans, form the important cells generally employed in the investigation of biological membranes, have some glycoproteins in their cell membrane. ${ }^{2}$ These membrane proteins are band 3 and glycophorins A-D, and some others glycoproteins. Glycophorin is a sialic acid conjugate with oligosaccharide which is rich in glycoproteins and it was detected by aid of staining with the periodic acid-Schiff's (PAS) reagent. ${ }^{6,7}$ There are 4 categories of glycophorins (A, B, C, and D) found on the extracellular part of the membrane, each with distinct function. ${ }^{8,9}$ Glycophorin A which is an important constituent of RBC membrane glycoproteins does form a dimeric state. ${ }^{3}$

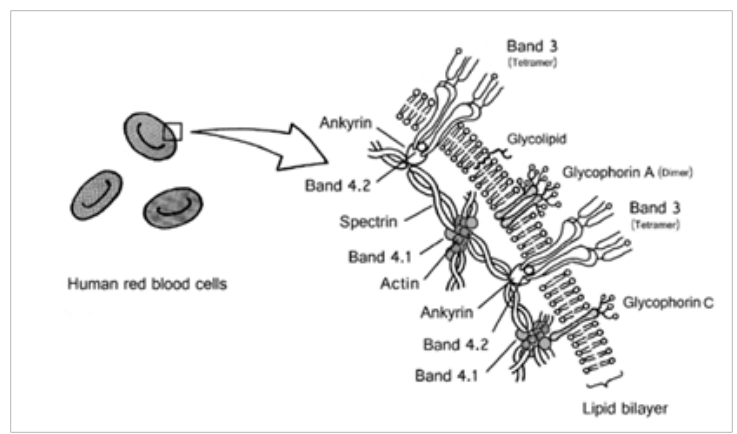

Figure I human red blood cell membranes. ${ }^{2}$

From the diagram above, band 3 has one $\mathrm{N}$-linked oligosaccharide, ${ }^{10}$ while glycophorins possess mostly O-linked oligosaccharides. Sialic acid (otherwise called $\mathrm{N}$-acetylneuraminic acid in the body of human) 
is seen at the extreme end of the O-linked oligosaccharide. Sialic acid (SA) bound to glycophorin A is responsible for the negative charge of the RBC membrane. ${ }^{2}$

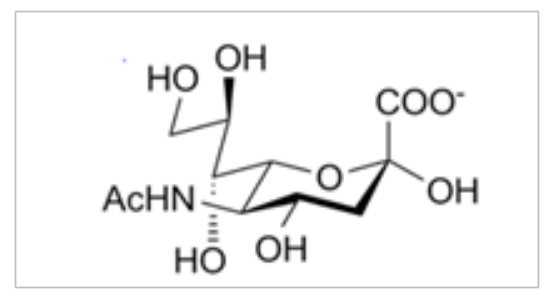

N-Acetylneuraminic acid (Sialic Acid or Neuraminic Acid). ${ }^{\prime \prime}$

Neuraminic acid is abundantly found in the cells of mammal and it forms complex with glycans (as found on mucins) and with glycoproteins, glycolipids, such as gangliosides which form an important component of neuronal membranes. This acid can be oxidized or cleaved from glyoproteins/glycolipids by neuraminidase. Sialic acid has a terminal sugar in glycoconjugates. ${ }^{2}$

Neuraminidase or sialidase enzyme cleaves sialic acid sugar moiety from selected glycoproteins (GP) and glycolipids (GL). The cleavage of GP or GL by NA promotes the release of trypanosome products into the cells.

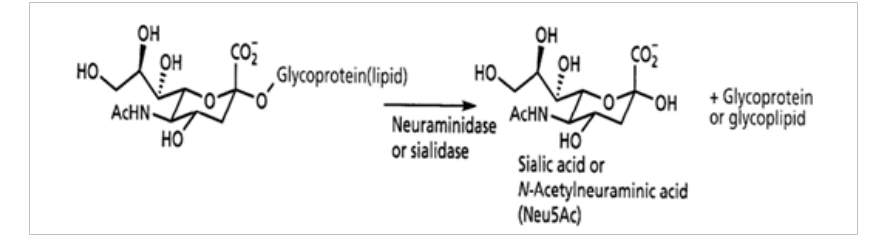

\section{Adhesion of trypanosome to red cell membrane}

Trypanosomes are describes to have the ability to cleave sialic acid from glycophorins on erythrocyte membranes with the aid of neuraminidase or sialidase. These parasites also make use of the erythrocyte sialoglycoproteins for their proliferation and differentiation. ${ }^{12}$

The event through which red blood cells become adhere to trypanosome has been demonstrated in vitro in T. gambiense, T. rhodesiense, T. congolense, T. brucei, T. lewisi and T. evansi infections. ${ }^{4,5}$ This occurrence has been experimented in vivo using transmission and scanning electron microscopy, which clearly displayed that the infected red blood cells may form cup-shape and may form tiny holes or breaches on the membranes of RBCs. ${ }^{4}$ The process of adhesion can lead to haemolysis of red cells (otherwise known as anaemia) which may be caused by the haemolytic factors such as haemolysin or high concentration FFA (such as linolenic acid) introduced into the red cells by the parasites. The haemolysis of red cells has been reported in T. congolense incubated red cells. ${ }^{4}$ In an experiment to determine the site where trypanosome bind on the cell membrane of red cell, RBCs were treated with neuraminidase, polyL-lysine, and sodium periodate which are compounds that can cleave, bind and oxidize sialic acid. Each of the compounds is able to block the binding of $T$. congolense to bovine red cells. The implication is that $\mathrm{RBC}$ sialic acid is the binding/adhesion site for trypanosome. The pre-treatment of the trypanosomes with trypsin and chymotrypsin also blocked the binding of $T$. congolense to the trypanosomes which usually adhere with their anterior sides. ${ }^{4,13}$

Furthermore, in the T. vivax infected cattle, RBC membrane sialic acid is depressed which become noticeable during the first wave of parasitaemia. This result can be interpreted as since trypanosome produced neuraminidase in vitro, it can also produce the enzyme in vivo which result in the cleavage of the surface sialic acid and even may be linked to the anaemic condition which is very prominent during the first wave of parasitaemia. ${ }^{4}$

\section{Leakage of vital components of RBC after infection}

The trypanosomes adhere to RBCs create breaches or holes through which cellular constituents leak into the extracellular space, thereby result in the abnormalities in the level of these biochemical parameters.

The level of red blood cell pyruvate kinase increased during infection of mice and rabbits by $T$. brucei, ${ }^{4}$ while in the $T$. vivax infected cattle, the levels d-amino levulinic acid is elevated. ${ }^{4}$ In the same vein, in the T. brucei infected mice, the levels of hexokinase, glucose-6-phosphate dehydrogenase, glutathione peroxidase, and glutathione reductase became increased. ${ }^{4}$

However, there is decrease in the levels of RBC potassium in $T$. brucei and T. equiperdum infected rat and this is thought to cause by the differential permeability of the RBC..$^{14}$ A report from a study showed that the plasma concentrations of the glycoproteins hexose, hexosamine, sialic acid and seromucoid fraction elevated in the cattle infected with $T$. brucei and T. vivax during the first eight weeks of infection when parasites appeared in the blood, but reduced from the tenth week when parasites had become very scanty. ${ }^{4}$ It was postulated from this study that the reduction in erythrocyte surface sialic acid was caused by its cleavage by trypanosome neuraminidase, and this was assumed to be responsible for destruction of erythrocytes early in infection and for the increase in serum sialic acid levels.

\section{Pathological anaemic shapes assumed by infected RBC}

RBCs cell membrane posed mechanical resistance to the trypanosomes before they are able to adhere successfully to the membrane and cause damages. The existence of many pathological form or shape assumed by RBC during the infection is confirmed by transmission and scanning electron microscopy. The several erythrocyte morphological abnormalities observed in trypanosomiasis infection of red blood cells include anisocytosis, poikilocytosis, macrocytosis, microcytosis, echinocytes (crenated erythrocytes), doughnut-shaped erythrocytes, spherocytosis and acantocytes have been describes, in various combinations, in $T$. brucei and $T$. congolence infections, ${ }^{4}$ and in human T. rhodesiense infection. This could also be the side effect of the redirected RBC antibodies which are adsorb into RBC by the anti-RBC antibodies synthesized by the parasite in invasion.

\section{Inhibitors of neuraminidase}

Neuraminidase inhibitors (NAIs) are a class of drugs which block the neuraminidase enzyme. 


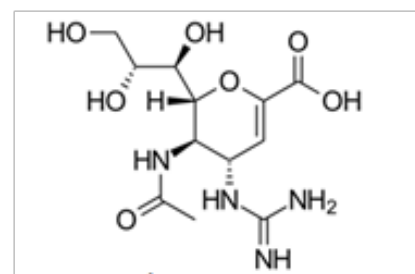

Oseltami

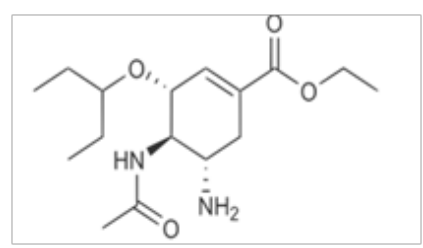

Zanamivir
These are used as antiviral drugs due to their potency to block the function of viral neuraminidases of the influenza virus. ${ }^{16}$ They perform this action by preventing its reproduction by budding from the host cell. The neuraminidase inhibitors oseltamivir and zanamivir were approved in the US and Europe for treatment and prevention of influenza A and B. ${ }^{13,15}$ These inhibitors which can bind to the active site of trypanosome neuraminidase thereby inhibit it, and eventually block the adhesion of the parasite to the cell membrane should be administered at the first wave of parasitaemia to reduce the damages done to the red blood cells by the infection and even the immune system.

\section{Conclusion}

There is need to fortify red blood cell membrane by inhibitors before the application of the necessary medications as it is the centre which receives first attack which result into massive destruction of the cells by the diseases and corresponding aftermath evacuation of wounded or dead cells by phagocytes.

\section{Recommendation}

Inhibitors of neuraminidase should be administered at the first wave of parasitaemia before administering the appropriate prescribed drug, to reduce the damages done to the red blood cells by the infection and even the immune system. More research work is needed to study the effectiveness of these inhibitors and their possible interactions with the immune system.

\section{Acknowledgments}

None

\section{Conflicts of interest}

The author declares that there are no conflicts of interest.

\section{References}

1. Taiwo VO, Olaniyi MO, Ogunsanmi AO. Comparative plasma biochemical changes and susceptibility of erythrocytes to in vitro peroxidation during experimental Trypanosome congolense and T brucei infections in sheep. J Isreal Vet Med Ass. 2003;58(4).
2. Takahiko Aoki. A comprehensive review of our current understanding of red blood cell (RBC) glycoproteins. Membranes (Basel). 2017;7(4):E56.

3. Smith SO, Eilers M, Song D, et al. Implications of threonine hydrogen bonding in the glycophorin A transmembrane helix dimer. $J$ Biophys. 2002;82:2476-2486.

4. Anosa VO. Haematological and biochemical in human and animal trypanosomisis Part I. Reve Elev Med Vet Pays Trop. 1988;41(2):151164.

5. Anosa VO, Kaneko JJ. Pathogenesis of T. brucei infections in deer mice (Peromyscus manicultus), Haematological, erythrocyte biochemical and iron metabolic aspects. Am J Vet Res. 1983;44(4):645- 651.

6. Fairbanks, G, Steck TL, Wallach DFH. Electrophoretic analysis of the major polypeptides of the human erythrocyte membrane. Biochemistry. 1971;10(13):2606-2617.

7. Doerner KC, White BA. Detection of glycoproteins separated by nondenaturing polyacrylamide gel electrophoresis using the periodic acid-Schiff stain. Anal Biochem. 1990;187(1):147-150.

8. Anstee DJ, Tanner MJA. Structure and function of the red cell membrane sialoglycoproteins. Br J Haematol. 1986;64(2):211-215.

9. Katunga-Rwakishaya E, Murray M, Holmes PH. Pathophysiology of Trypanosoma congolense infection in two breeds of sheep, Scottish blackface and Finn. Dorset Vet Parasitol. 1997;68(3):215-225.

10. Tanner MJA. Molecular and cellular biology of the erythrocyte anion exchanger (AE 1). Semin Hematol. 1993;30(1);34-57.

11. Severi E, Hood DW, Thomas GH. Sialic acid utilization by bacterial pathogens. Microbiology. 2007;153(9):2817-2822.

12. Owens JW, Mueller TJ, Morrison M. A minor sialoglycoprotein of the human erythrocyte membrane. Arch Biochem Biophys. 1980;204:247254.

13. Jefferson Tom, Jones Mark, Doshi Peter, et al. Oseltamivir for influenza in adults and children: systematic review of clinical study reports and summary of regulatory comments. BMJ. 2014;348:2545.

14. Bradbury MWB, Stubbs J, Hughes IE, et al. The distribution of potassium, Sodium, chloride and urea between lumbar CSF and blood brain barrier in human Subjects. Clin Sci. 1963;25:97-105.

15. Heneghan Carl J, Onakpoya Igho, Thompson Matthew, et al. Zanamivir for influenza in adults and children: systematic review of clinical study reports and summary of regulatory comments. BMJ. 2014;348:g2547.

16. Sugaya Norio. Widespread use of neuraminidase inhibitors in Japan. $J$ Infect Chemother. 2011;17(5):595-601. 